\title{
LCMS/MS and TOF-SIMS Identification of the Color Bodies on the Surface of a Polymer
}

\author{
Colin Moore \\ Crompton Corporation, Middlebury, Connecticut, USA
}

\author{
Pat McKeown \\ Evans East, East Windsor, New Jersey, USA
}

\begin{abstract}
The source of discoloration on a polymer surface can often be identified by washing the surface of the discolored polymer to collect the color bodies, then analyzing the washings using liquid chromatography-mass spectrometry (LCMS), with an in-line ultraviolet (UV) detector set at the optimum wavelength for the particular color bodies. A reference sample having no discoloration is also analyzed in the same way. In this paper, results from this methodology are compared with direct time of flight-secondary ion mass spectrometry (TOF-SIMS) analysis of a discolored polymer. The benefits and shortcomings of each methodology are discussed. (J Am Soc Mass Spectrom 2005, 16, 295-301) (C) 2004 American Society for Mass Spectrometry
\end{abstract}

S urface discoloration of a polymer can result from a number of different phenomena, e.g., contamination, component migration, oxidation, and other chemical reactions. Components rising to the surface can give rise to bloom [1], e.g., protective waxes are beneficial, but thiazoles (Mercaptobenzothiazole) are undesirable. Oxidation of antioxidants can form color bodies, e.g., phenolic antioxidants can form quinone methides [2]. The first step in identifying a color body is to separate it from the polymer, and this can often be achieved by washing the surface with a suitable solvent. The washings will be a complex mixture of the color bodies, additives, and other surface contaminants. Identification of the colored components requires further separation of the mixture, analysis of the separated components, and the ability to ascertain which of the components are colored. The combination of LCMS(/MS) with an in line diode array detector (DAD) is able to do the complete analysis in a single experiment.

The introduction of atmospheric pressure ionization sources has transformed LCMS from a difficult and unreliable technique into a routine analytical tool [3]. Color bodies are often polar molecules, which means that they are easy to ionize in an electrospray (ESI) ion source. However, the electrospray spectrum contains few (if any) fragment ions and therefore the identifica-

Published online January 14, 2005

Address reprint requests to Dr. C. Moore, Crompton Corporation, 199 Benson Road, Mail Stop G-20, Middlebury, CT 06749, USA. E-mail: colin_moore@cromptoncorp.com tion of unknowns requires that either an LCMS/MS spectrum is acquired and/or exact mass measurements [4] are performed to get the elemental formula of the pseudo molecular ion. An in line UV detector (preferably a DAD) is employed to determine which of the components separated by the HPLC column absorb at the appropriate wavelength to give the observed discoloration. Structure elucidation is made much easier if the analyst has a thorough knowledge of the sample chemistry and it's history.

Imaging mass spectrometry [5] is a powerful technique for mapping the concentration of a compound on the surface of a matrix and has applications in many fields, including the analysis of inorganic materials, polymers, and biological materials. If the mass spectrometer is a time of flight (TOF) system, and is used to mass analyze secondary ions produced by bombarding a surface with high-energy particles, the technique is known as TOF-SIMS [6]. TOF-SIMS has been used to detect light stabilizers [7] and antioxidants [8] on the surface of a polymer, as well as for the characterization of the bulk polymer [9].

The polymer formulations in this study all contain mixtures of benzothiazole and thiuram disulfide accelerators, which are known to be synergistic combinations $[10,11]$.

\section{Experimental}

The outermost layer of normal and discolored polymer samples were washed with acetonitrile and the washings 
Table 1. Compounds of interest in the 3 polymer layers

\begin{tabular}{|c|c|c|c|}
\hline \multirow[b]{2}{*}{ Component } & \multicolumn{3}{|c|}{ Amount (\%) } \\
\hline & Bottom Layer & Middle Layer & Top Layer \\
\hline EPDM & 38 & 33 & 33 \\
\hline N650 - Thermal process Carbon Black & 33 & - & - \\
\hline N990 - Thermal process Carbon Black & 11 & - & - \\
\hline Sunpar 2280 - high viscosity, low volatility paraffinic oil & 12 & 6 & 6 \\
\hline CMBT - Copper 2-Mercaptobenzothiazolate & $<1$ & - & - \\
\hline Clay & - & 38 & 38 \\
\hline Hisil 532 - Precipitated Silica inorganic filler & - & 10 & 10 \\
\hline $\mathrm{CaCO}_{3}$ & - & 5 & 5 \\
\hline TBTD - Tetrabutyl thiuram disulfide & 1 & $<1$ & $<1$ \\
\hline Black masterbatch containing ODPA - Octylated & & & \\
\hline diphenylamine & - & $<1$ & $<1$ \\
\hline
\end{tabular}

evaporated to low volume. It was noted that after washing, the discolored sample lost its color, and its washings were a pale yellow/green in color, whereas the washings from the control sample were colorless.

\section{LCMS and LCMS/MS}

Spectra were acquired on a Hewlett-Packard (Palo Alto, CA) 1090 series II liquid chromatograph with a diode array detector, coupled to a Micromass (Beverly, MA) Q-TOF2 mass spectrometer. Chromatography was performed on a $2.1 \mathrm{~mm}$ diameter by $150 \mathrm{~mm}$ Luna RP column (Phenomenex, Torrance, CA) eluted with a mobile phase consisting of water (A) and acetonitrile (B). A gradient elution at $300 \mathrm{uL} / \mathrm{min}$ starting from $70 \%$ A to $5 \% \mathrm{~A}$ in $10 \mathrm{~min}$, with a $10 \mathrm{~min}$ hold at $5 \%$ A was used to separate the compounds of interest prior to mass spectral analysis. DAD/UV spectra were collected at $2 \mathrm{~nm}$ resolution. The Q-TOF was operated in positive ion electrospray mode with $3.0 \mathrm{kV}$ applied to the inlet capillary. The LM and HM resolutions on the quadrupole filter were set to 5, resulting in a bandwidth of $\sim 1.5 \mathrm{Da}$. and the inlet cone was set at $100 \mathrm{~V}$. Two product ion experiments of $471^{+}$were performed, one at $80 \mathrm{~V}$ collision energy, and a second at $40 \mathrm{~V}$, using argon as the collision gas. Prior to the analysis, the TOF analyzer was calibrated with $\mathrm{NaI} / \mathrm{RbI}$ to $10 \mathrm{ppm}$ accuracy.

\section{TOF-SIMS}

Spectra were acquired using a Physical Electronics model TFS-2000 TOF-SIMS (Eden Prairie, MN). The primary ion source was gallium. The liquid metal source was operated at a beam energy of $18 \mathrm{kV}$, a repetition rate of $\sim 10 \mathrm{kHz}$ and a pulse width of $\sim 1 \mathrm{~ns}$. The beam was rastered over a $256 \times 256$ pixel area (150 $\mu \mathrm{m} \times 150 \mu \mathrm{m}$ area). The gun was fired at each pixel and a full mass spectrum was recorded. The beam position was recorded with each spectrum allowing for chemical maps to be extracted from the data set. A low-energy $(<30 \mathrm{eV})$ electron source was utilized (pulsed alternately with the primary ion source) to minimize static charge build-up on the electrically insulating samples. Positive secondary ions were extracted from the surface after each primary ion pulse. These secondary ions were accelerated into a triple focusing time-of-flight mass analyzer. The secondary ions were detected using a micro-channel plate detector and a 256 stop time-todigital converter (156 ps time resolution). The flight time for each ion packet was recorded and the spectrum converted to a mass scale using a time to mass algorithm built into the instrument software.

\section{Results and Discussion}

The polymer samples were three component experimental E/P formulations. Each layer was a compounded ethylene/propylene/diene monomer (EPDM) rubber containing filler, carbon black, plasticizer, and antioxidants (Table 1); on aging the top layer of the polymer became discolored.

Figure 1 contains the HPLC results from the diode array detector for the analysis of the acetonitrile washings of the colored and control polymers. The UV chromatograms show that the extract from the discolored sample contains one component (retention time 13.28 minutes) that absorbs strongly over the range 380 to $450 \mathrm{~nm}$, and is not present in the reference sample. The complete UV spectrum and electrospray mass spectrum of this component are in Figure 2.

The molecular weight and isotopic distribution of the molecular ion are consistent with it being tetrabutyl thiuram disulfide (TBTD) plus copper (Figure 3). Smith et al. [12], reported that copper ions could form two different types of complexes with thiuram disulfides. An $\mathrm{ML}^{+}$complex, which is water-soluble and has a $\lambda_{\max }$ near $380 \mathrm{~nm}$ and a neutral $\mathrm{ML}_{2}$ complex with $\lambda_{\max }$ around $430 \mathrm{~nm}$. The UV spectrum of the complex detected in the acetonitrile washings is consistent with it having the $\mathrm{ML}_{2}$ structure. 

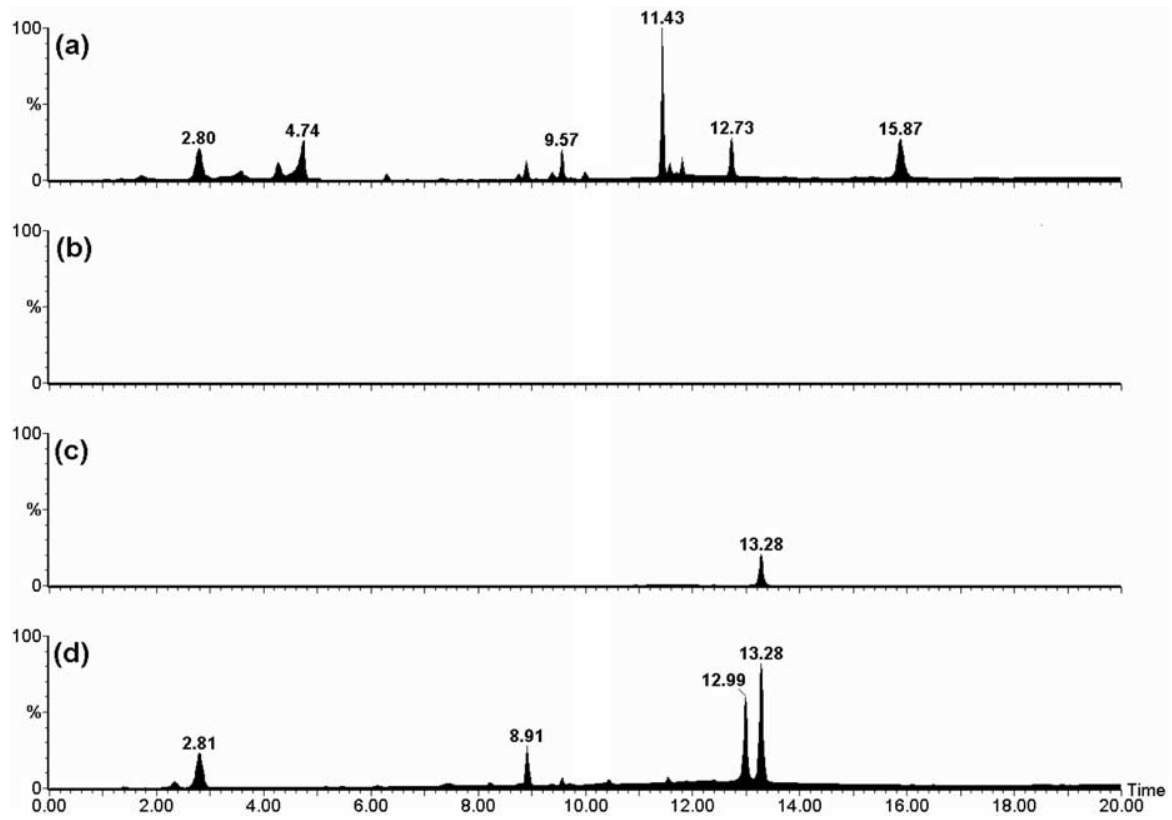

Figure 1. DAD traces from the LCMS analysis of the acetonitrile extracts: (a) Extract from control polymer, 220 to $500 \mathrm{~nm}$; (b) extract from control polymer, 380 to $450 \mathrm{~nm}$; (c) extract from Green polymer, 380 to $450 \mathrm{~nm}$; (d) extract from Green polymer, 220 to $500 \mathrm{~nm}$.

This neutral complex will only be detected if it becomes charged in the electrospray source. Positive ion electrospray mass spectra usually contain $(\mathrm{M}+$ $\mathrm{X})^{+}$ions, where $\mathrm{X}$ is, e.g., $\mathrm{H}, \mathrm{Na}$, or $\mathrm{NH}_{4}$. Metal complexation has been used to improved the sensitivity of LCMS, e.g., Brodbelt et al. [13] report that when flavanoids are complexed with a metal and neutral auxiliary ligand (L) they form cationized species that are two orders of magnitude more abundant than the protonated species, but the resulting ion is $\left[\mathrm{M}^{\mathrm{II}} \text { (flavonoid-H)L }\right]^{+}$.

Traeger has reported [14] that the neutral metal carbonyl compound cis- $\mathrm{Cr}(\mathrm{CO})_{2}(\text { dpe })_{2}$ (dpe = $\left.\mathrm{Ph}_{2} \mathrm{PCH}_{2} \mathrm{CH}_{2} \mathrm{PPh}_{2}\right)$; gives an ESI spectrum containing

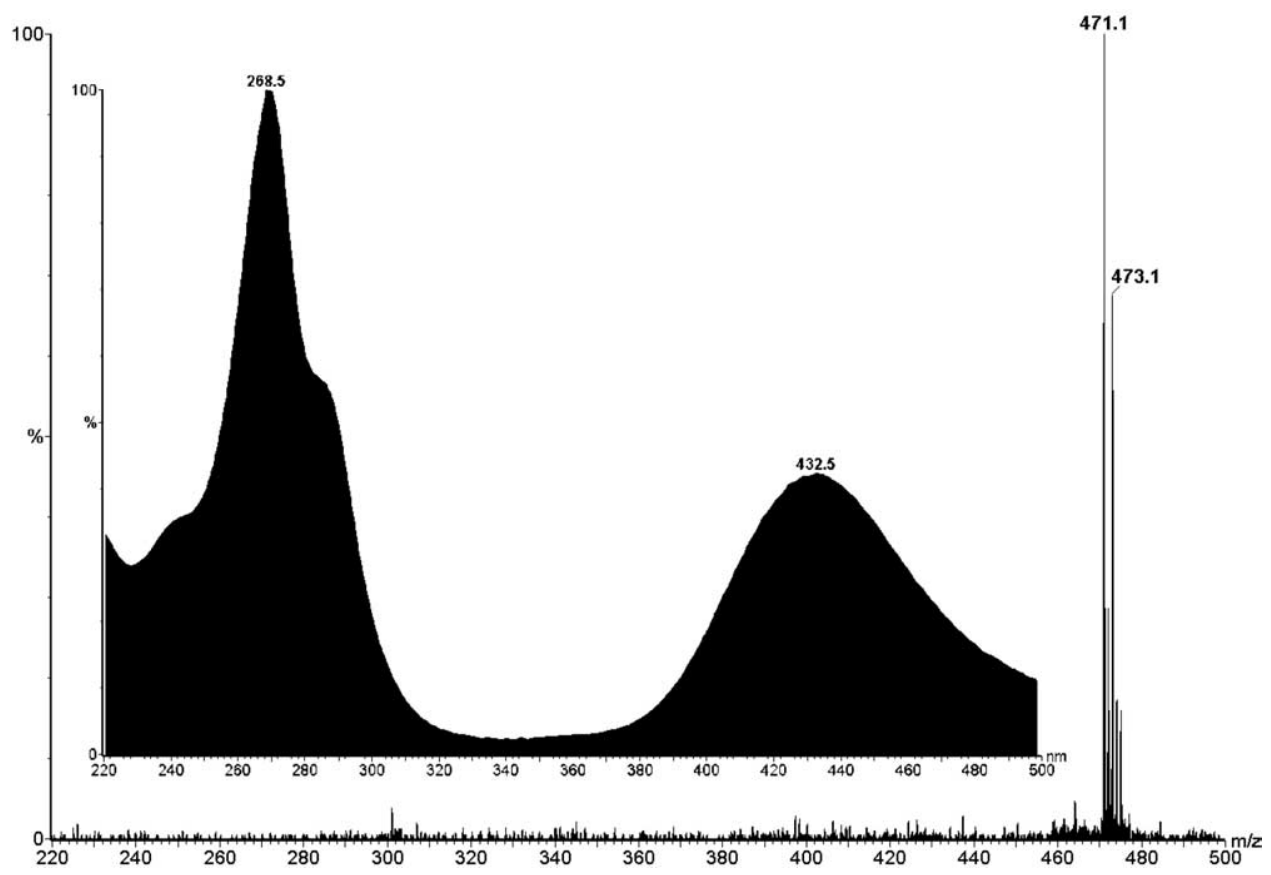

Figure 2. LCMS spectrum and UV spectrum of the component in the discolored polymer extract that elutes from the HPLC column at $13.28 \mathrm{~min}$. 

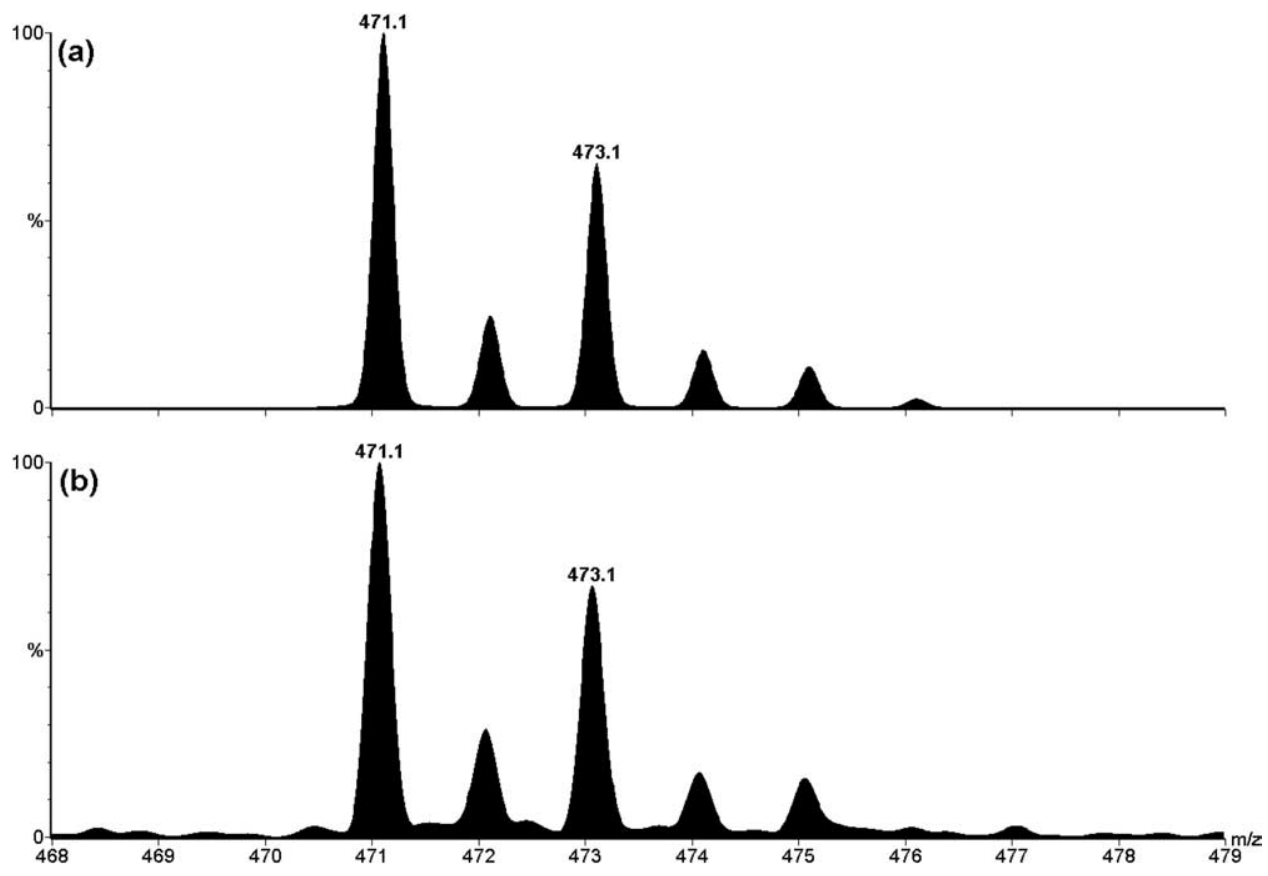

Figure 3. Isotope distributions: (a) Theoretical distribution for $\mathrm{C}_{18} \mathrm{H}_{36} \mathrm{~N}_{2} \mathrm{~S}_{4} \mathrm{Cu}$; (b) acquired data for the color body.

the 17-electron trans- $\left[\mathrm{Cr}(\mathrm{CO})_{2}(\mathrm{dpe})_{2}\right]^{+}$cation, i.e., $\mathrm{Cr}(0)$ has been oxidized to $\mathrm{Cr}(\mathrm{I})$. Dithiocarbamate ligands are known to stabilize high oxidation states [15], i.e., $\mathrm{Cu}(\mathrm{III})$ in<smiles>CCCCCC[N+](=C([O-])[O-])c1ccccc1</smiles>

Scheme 1

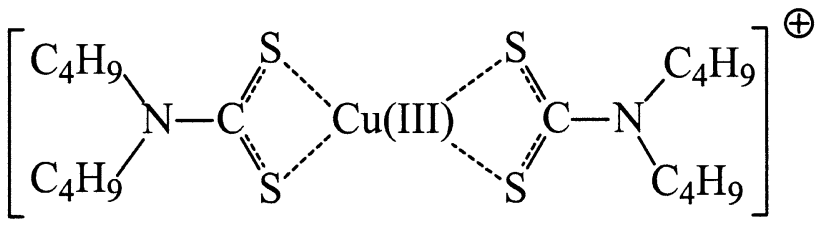

Scheme 2
$\left[\mathrm{Cu}\left(\mathrm{R}_{2} \mathrm{dtc}\right)_{2}\right]^{+}$. They are able to do this because they have a resonance form $\mathbf{1}$, that has a positive charge on the nitrogen and the $\pi$-electron flow from nitrogen to the sulfur atoms gives a ligand with very strong $\sigma$-donor properties.

We conclude, therefore, that the color body is the neutral $\mathrm{ML}_{2}$ complex, and that in the electrospray source copper (II) is being oxidized to copper (III). The resulting cation is stable because $\mathrm{L}$ is a dithiocarbamate. Reduction of $\mathrm{Cu}(\mathrm{II})$ complexes during electrospray has been reported by Gianelli et al. [16].

The electrospray LCMS spectrum contains few fragment ions and therefore an LCMS/MS spectrum was obtained as shown in Figure 4, and the fragment ions in the LCMS/MS spectrum are consistent with the suggested structure. When the mass calibration of the MS/MS spectrum was corrected to give the expected mass for the $\mathrm{m} / \mathrm{z} 116$ fragment ion, then the results listed in Table 2 were obtained. These fragment ion masses are in good agreement with the

Table 2. Masses and most likely elemental compositions of the fragment ions in the $\mathrm{ms} / \mathrm{ms}$ spectrum (Figure 4)

\begin{tabular}{|c|c|c|c|c|}
\hline $\begin{array}{l}\text { Measured } \\
\text { mass }(\mathrm{Da})\end{array}$ & $\begin{array}{c}\text { Relative } \\
\text { abundance (\%) }\end{array}$ & Calculated mass (Da) & $\begin{array}{c}\text { Measured-calculated } \\
\text { mass }(\mathrm{mDa})\end{array}$ & Formula \\
\hline 116.0354 & 36.13 & 116.0354 & 0 & $\mathrm{C}_{5} \mathrm{H}_{10} \mathrm{NS}$ \\
\hline 128.1436 & 10.46 & 128.1439 & -0.3 & $\mathrm{C}_{8} \mathrm{H}_{18} \mathrm{~N}$ \\
\hline 172.1165 & 100 & 172.1160 & 0.5 & $\mathrm{C}_{9} \mathrm{H}_{18} \mathrm{NS}$ \\
\hline 190.0668 & 1.68 & 190.0657 & 1.1 & $\mathrm{C}_{8} \mathrm{H}_{17} \mathrm{NCu}$ \\
\hline 268.0255 & 20.36 & 268.0255 & 0 & $\mathrm{C}_{9} \mathrm{H}_{19} \mathrm{NS}_{2} \mathrm{Cu}$ \\
\hline 286.0357 & 3.57 & 286.0361 & -0.4 & $\mathrm{C}_{9} \mathrm{H}_{21} \mathrm{ONS}_{2} \mathrm{Cu}$ \\
\hline 395.1639 & 1.08 & 395.1649 & -1 & $\mathrm{C}_{20} \mathrm{H}_{31} \mathrm{~N}_{2} \mathrm{~S}_{3}$ \\
\hline 471.1102 & 63.13 & 471.1057 & 4.5 & $\mathrm{C}_{18} \mathrm{H}_{36} \mathrm{~N}_{2} \mathrm{~S}_{4} \mathrm{Cu}$ \\
\hline
\end{tabular}




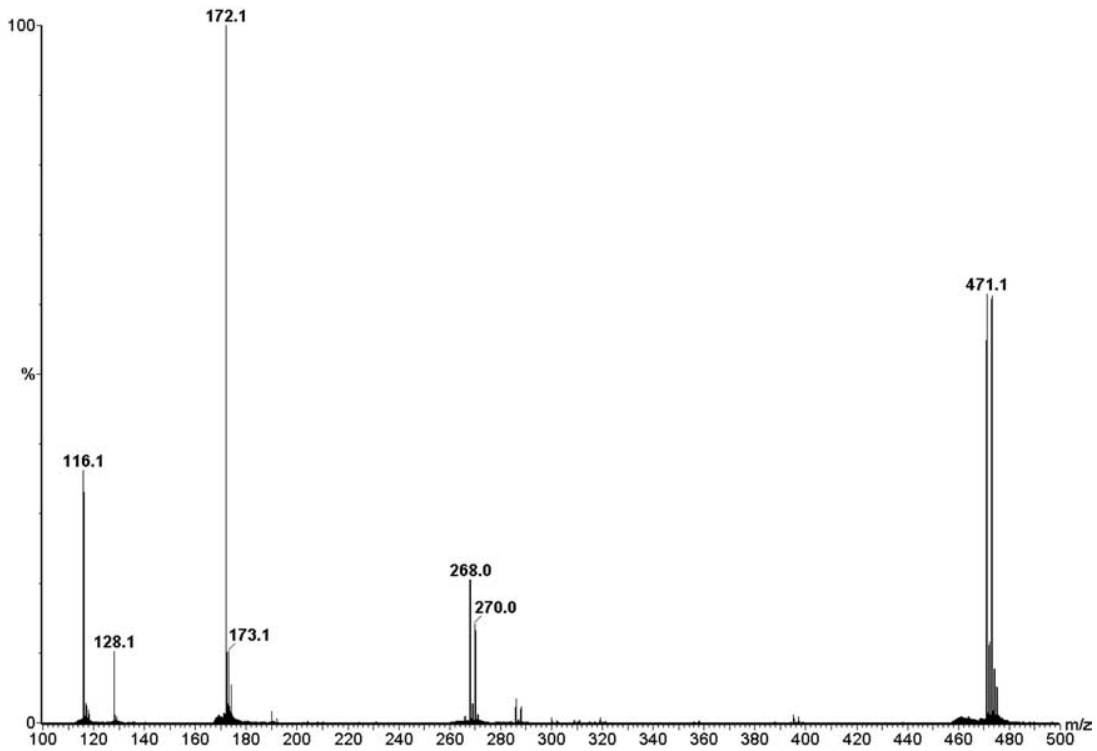

Figure 4. LCMS/MS spectrum of the pseudo molecular ion $\mathrm{m} / \mathrm{z}$ 471. The exact masses and most likely elemental compositions for these ions are in Table 2.

calculated masses for the expected fragment ions. The only anomalous result is that for the fragment ion at $\mathrm{m} / \mathrm{z} 286$, because the exact mass gives an elemental formula containing oxygen.

The strong affinity between metals and dithiocarbamates is well documented in the scientific literature, and forms the basis of a number of analytical methods for the quantitative determination of metals. Sedlak et al. [17] report that for complexes of metals and Diethyldithiocarbamates the complexing strength follows the order: $\mathrm{Cu}(\mathrm{II})>\mathrm{Ni}$ (II) $>\mathrm{Pb}$ (II) $>\mathrm{Cd}$ (II) $>\mathrm{Zn}$ (II).

A similar relationship is not unexpected for the dibutydithiocarbamate, which may explain why copper from the copper 2-mercaptobenzothiazolate (CMBT) in the bottom layer migrates through the upper two lay- ers, and forms the green complex with the tetrabutyl thiuram disulfide (TBTD) in the top layer. More details of the diffusion of curatives in elastomers can be found in studies by Gardiner [18, 19].

Having identified the source of discoloration using extraction plus LCMS/MS, we wondered whether similar data could be obtained by direct analysis of the polymer surface. Survey spectra were obtained from both the outside surface of the polymer and by slicing the polymer and looking at the freshly exposed polymer surface. TOF-SIMS of the surface of the discolored sample yielded spectra (Figure 5a and c) that contained the $\mathrm{m} / \mathrm{z} 471$ pseudo molecular ion and $\mathrm{m} / \mathrm{z} 172$ fragment ion that were seen in the electrospray LCMS/MS spectra (Figure 4), and these ions were absent in the spec-
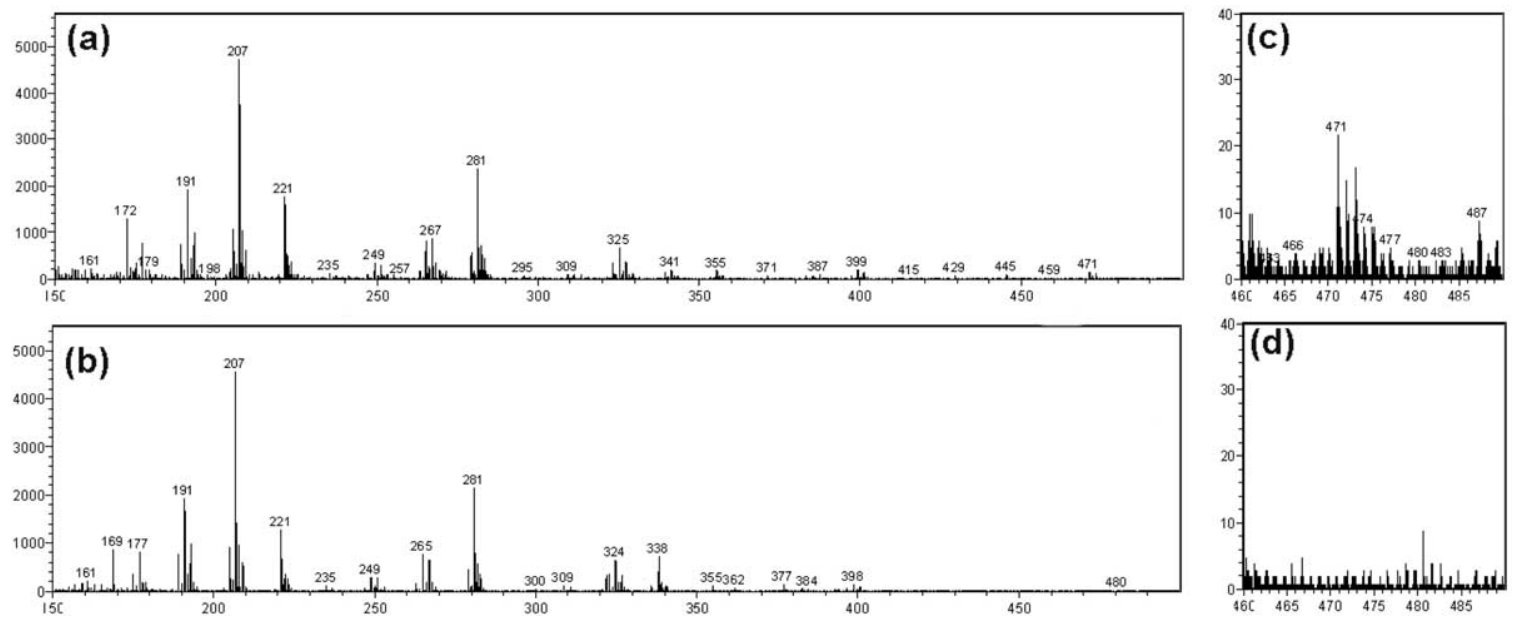

Figure 5. TOF-SIMS spectra of the polymer surface: (a) Discolored polymer; (b) the control sample; (c) expansion of discolored polymer spectrum to show the isotopic distribution of the $\mathrm{m} / \mathrm{z} 471$ pseudo molecular ion; (d) expansion of control polymer to show the absence of the $\mathrm{m} / \mathrm{z} 471$ pseudo molecular ion. 

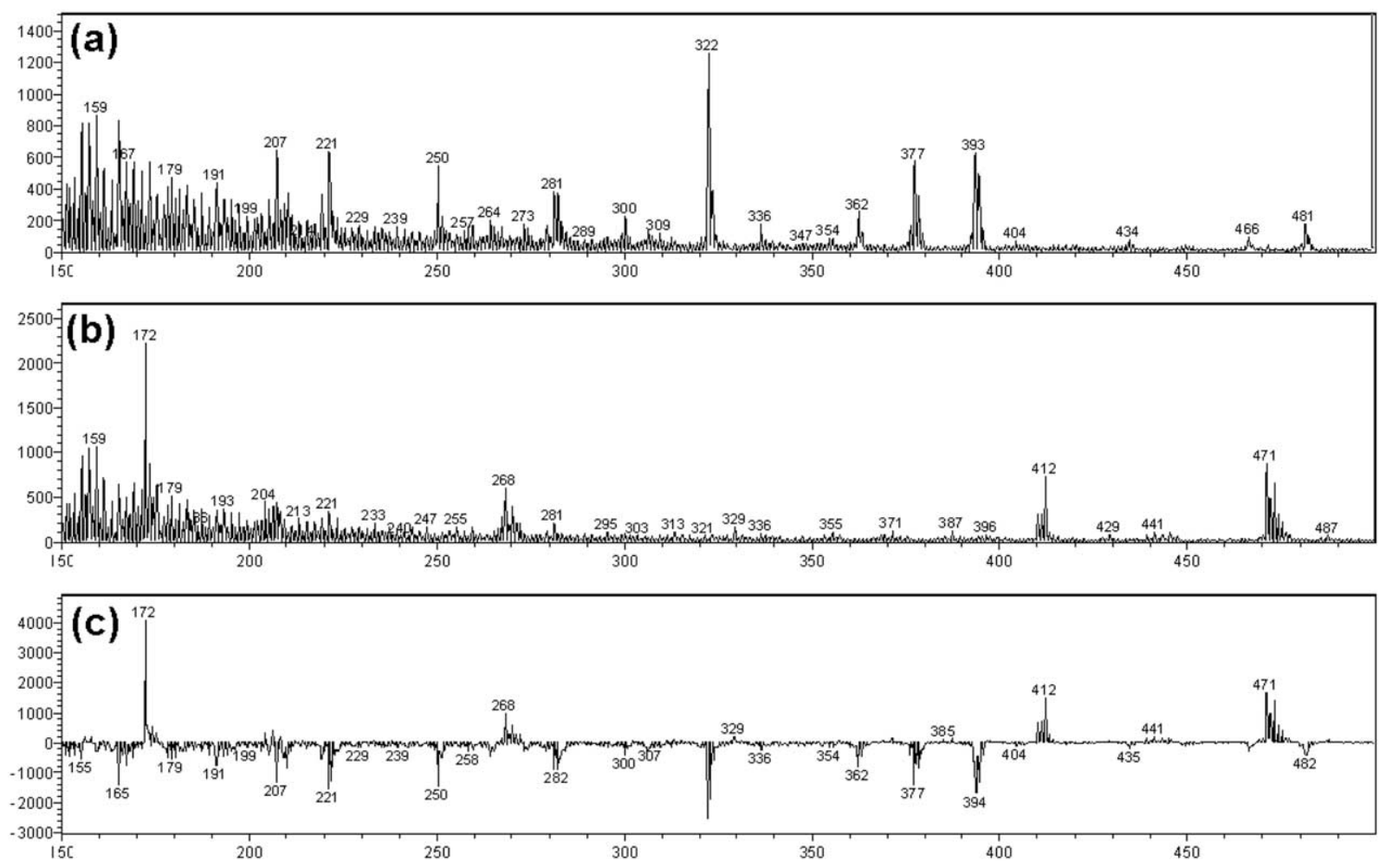

Figure 6. (a) Spectrum of the control polymer; (b) spectrum of the discolored sample when both spectra were acquired from freshly exposed polymer; (c) Spectrum a subtracted from Spectrum $\mathbf{b}$.

trum of the control sample (Figure $5 b$ and $d$ ). The spectra of both samples contain many ions from silicones, e.g., $\mathrm{m} / \mathrm{z} 207,281$, and 325 which are common surface contaminents, that make it difficult to see the ions of interest. The relative abundance of these ions was reduced when spectra were obtained from a freshly exposed polymer surface (Figure $6 a$ and $b$ ), and because the color body was distributed throughout the upper polymer layer. The same conclusions were reached from the two methods of sample preparation. By subtracting the averaged spectrum of the control sample from that of the discolored sample (Figure 6c), more fragment ions indicative of the presence of the copper dithiocarbamate were detected, i.e., $\mathrm{m} / \mathrm{z} 412$ and 268 . If

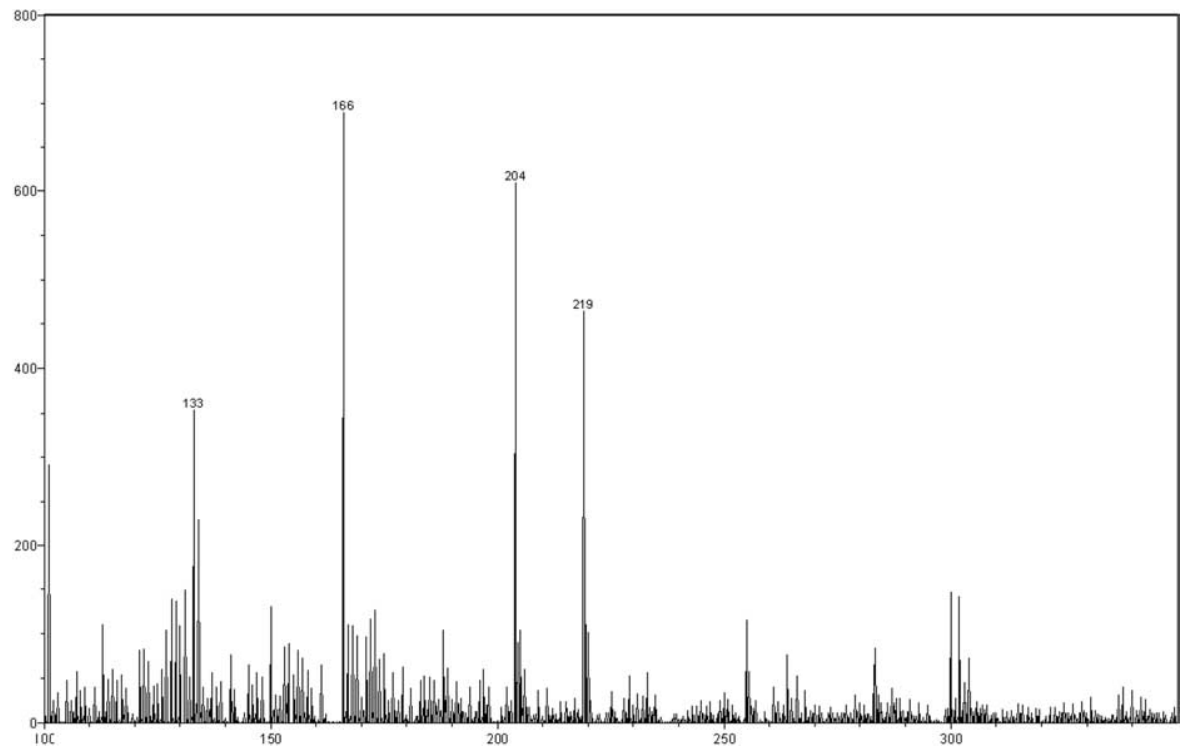

Figure 7. Negative ion TOF-SIMS spectrum of the control polymer subtracted from the spectrum of the discolored sample. 
one compares Figures 6 and 5 with Figure 4, then it is apparent that the copper dithiocarbamate is only detected in the colored sample, and that direct TOF-SIMS analysis is giving the same results as the more complex extraction followed by LCMS/MS methodology.

Close examination of the spectra of the surface showed the presence of ions from oxidation of the thiuram $(m / z 487$ Figure $5 c)$ that are not present in the LCMS spectra. The $m / z 394$ ion in the spectrum of the control sample is protonated octylated diphenylamine (ODPA) antioxidant (also detected by GCMS analysis of the extracts) and the amine gives the expected $\mathrm{MH}^{+}$ion [20]. The background subtracted negative ion SIMS spectra (Figure 7) were interesting because they contained benzothiazole ions from the CMBT $(\mathrm{m} / \mathrm{z} 166)$ and fragment ions from the thiuram $(m / z 204)$. One can also see evidence for stearate $(m / z 283)$, palmitate $(m / z 255)$, and myristate $(\mathrm{m} / \mathrm{z} 227)$ ions coming from stearic acid in the polymer.

\section{Conclusions}

The method of choice for the analysis of color bodies on the surface of a polymer is obviously extraction followed by LCMS/MS with an in-line DAD, because this gives unequivocal identification of the colored components. However, the method is time consuming and labor intensive. This study shows that TOF-SIMS can quickly provide clues as to the identity of color bodies, which for the analysis of known formulations may provide enough information to allow reformulation of the polymer so as to avoid the discoloration problem. For unknown formulations, the data analysis can be more complicated, as current TOF-SIMS databases are limited compared to the more extensive electron impact ionization MS databases available. However, the high mass resolution data provided by TOF-SIMS can be used to create a short list of possible fragments that can be useful for spectral interpretation. TOF-SIMS can also provide information about oxidation of compounds on the surface, which is not readily detected when samples are analyzed using the extraction LCMS methodology. Finally, these results suggest that once a color body has been identified, TOF-SIMS can be used to quickly confirm that the same color body causes surface discoloration of new samples.

\section{Acknowledgments}

The authors gratefully acknowledge $\mathrm{Al}$ Kind at the University of Connecticut for acquiring the LCMS/MS spectra, and Xia Dong at Evans East for acquiring the initial TOF-SIMS spectra.

\section{References}

1. Kuzminskii, S.; Feldshtein L. S.; Reitlinger S. A. Influence of Mechanical Stress on Aging of Vulcanized Rubbers. Rubber Chem. Tech. 1962, 35, 147.
2. Pospíšil, J.; Habicher, W.-D.; Pilař, J.; Nešpůrek, S.; Kuthan, J.; Piringer, G.-O.; Zweifel, H. Discoloration of Polymers by Phenolic Antioxidants. J. Polym. Degrad. Stab. 2002, 77, 531.

3. In Applied Electrospray Mass Spectrometry, 2nd ed.; Pramanik, N.; A. K. Ganguly, A. K.; Gross, M. L., Eds.; Marcel Dekker: New York 2002.

4. Maizels, M.; Budde, W. L. Exact Mass Measurements for Confirmation of Pesticides and Herbicides Determined by Liquid Chromatography/Time-of-Flight Mass Spectrometry. Anal. Chem. 2001, 73, 5436.

5. Pacholski, M. L.; Winograd, N. Imaging with Mass Spectrometry. Chem. Rev. 1999, 99, 2977.

6. Blestos, I. S.; Hercules, D. M.; van Leyen, D.; Hagenhoff, B.; Niehuis, E.; Benninghoven, A. Molecular Weight Distributions of Polymers Using Time-of-Flight Secondary-Ion Mass Spectrometry. Anal. Chem. 1991, 63, 1953.

7. Andrawes, F.; Valcarcel, T.; Haacke, G.; Brinen, J. Depth Distribution of Light Stabilizers in Coatings Analyzed by Supercritical Fluid Extraction-Gas Chromatography and Time-of-Flight Secondary Ion Mass Spectrometry. Anal. Chem 1998, 70, 3762.

8. Walzak, M. J.; McIntyre, N. S.; Prater, T.; Kaberline, S.; Graham, B. A. Detection and Mapping of Chimassorb 944FD Antioxidant Additive in Polyethylene Using TOF-SIMS. Anal. Chem. 1999, 71, 1428.

9. Briggs, D.; Fletcher, I. W.; Reichlmaier, S.; Agulo-Sanchez, L. J. Short, R. D. SIMS Applied to Polymer Surfaces. Part 18. Surface Morphology of a PVC/PMMA Blend Studied by TOF-SIMS. Surf. Interface Anal. 1996, 24, 419.

10. Debnath, S. C.; Basu, D. K. Studies on the Effect of Thiuram Disulfide on NR Vulcanization Accelerated by Thiazole-Based Accelerator Systems. J. Appl. Polym. Sci. 1996, 60, 845.

11. Chaser, D. W. Tetraisobutylthiuram Monosulfide: How can it be both a Cure Retarder and Accelerator with Benzothiazole Sulfenamides? Rubber Chem. Tech. 1997, 70, 634.

12. Smith, R. M.; Morarji, R. L.; Salt, W. G. Determination of Dithiocarbamates by Liquid Chromatography Using Transition-Metal Salts as "Ion-Pair" Reagents. Analyst 1981, 106, 129.

13. Satterfield, M.; Brodbelt, J. S. Enhanced Detection of Flavonoids by Metal Complexation and Electrospray Ionization Mass Spectrometry. Anal. Chem. 2000, 72, 5898.

14. Traeger, J. C. Electrospray Mass Spectrometry of Organometallic Compounds. Int. J. Mass Spectrom. 2000, 200, 387.

15. Bond, A. M.; Martin, R. L. Electrochemistry and Redox Behavior of Transition Metal Dithiocarbamates. Coord. Chem. Rev. 1984, 54, 23.

16. Gianelli, L.; Amendola, V.; Fabbrizzi, L.; Pallavicini, P.; Mellerio, G. G. Investigation of Reduction of $\mathrm{Cu}$ (II) Complexes in Positive-Ion Mode Electrospray Mass Spectrometry. Rapid Commun. Mass Spectrom. 2001, 15, 2347.

17. K. W. Weissmahr, K. W.; Houghton, C. L.; Sedlak, D. L. Analysis of the Dithiocarbamate Fungicides Ziram, Maneb, and Zineb and the Flotation Agent Ethylxanthogenate by Ion-Pair Reversed-Phase HPLC. Anal. Chem. 1998, 70, 4800.

18. Gardiner, J. B. Curative Diffusion Between Dissimilar Elastomers and its Influence on Adhesion. Rubber Chem. Tech. 1968, 41, 1312.

19. Gardiner, J. B. Measurement of Curative Diffusion between Rubbers by Microinterferometry. Rubber Chem. Tech. 1969, 42, 1058.

20. In Secondary Ion Mass Spectrometry; Benninghoven, A.; Ruedenauer, F. G.; Werner, H. W., Eds.; John Wiley and Sons: New York, 1987. 